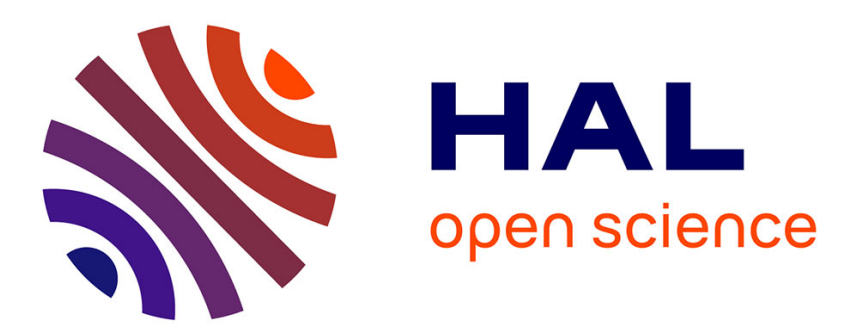

\title{
Analyse pour le calage de modèles hydrauliques à surface libre: une approche par les théories des systèmes linéaires et de l'automatique
} P.O. Malaterre, J.P. Baume, N. Jean-Baptiste, J. Sau

\section{To cite this version:}

P.O. Malaterre, J.P. Baume, N. Jean-Baptiste, J. Sau. Analyse pour le calage de modèles hydrauliques à surface libre: une approche par les théories des systèmes linéaires et de l'automatique. La Houille Blanche - Revue internationale de l'eau, 2011, 3, p. 63 - p. 68. 10.1051/lhb/2011034 . hal-00620623

\section{HAL Id: hal-00620623 \\ https://hal.science/hal-00620623}

Submitted on 8 Sep 2011

HAL is a multi-disciplinary open access archive for the deposit and dissemination of scientific research documents, whether they are published or not. The documents may come from teaching and research institutions in France or abroad, or from public or private research centers.
L'archive ouverte pluridisciplinaire HAL, est destinée au dépôt et à la diffusion de documents scientifiques de niveau recherche, publiés ou non, émanant des établissements d'enseignement et de recherche français ou étrangers, des laboratoires publics ou privés. 


\title{
Analyse pour le calage de modèles hydrauliques à surface libre : une approche par les théories des systèmes linéaires et de l'automatique
}

\author{
Pierre-Olivier MALATERRE ${ }^{1}$, Jean-Pierre BAUME ${ }^{1}$, Nelly JEAN-BAPTISTE ${ }^{1}$, Jacques SAU²
}

\author{
1. UMR G-EAU, CEMAGREF, 361 rue Jean-François Breton, B.P. 5095, 34196 Montpellier Cedex 05, France - e-mail : pierre-olivier.mala- \\ terre@cemagref.fr, jean-pierre.baume@cemagref.fr,nelly.jean-baptiste@cemagref.fr \\ 2. LMFA UMR 5509, Université de Lyon 1, 43 Bd du 11 Novembre 1918, 69622 Villeurbanne Cedex, France-e-mail : jacques.sau@univ-lyon1.fr
}

\begin{abstract}
RÉSUMÉ. - Tout projet mettant en œuvre un modèle nécessite une phase de calage. Pour un modèle hydrodynamique basé sur les équations de Saint-Venant, cela signifie ajuster des coefficients de frottement, des coefficients de débits aux ouvrages frontaux et latéraux, et des termes d'infiltration. Plusieurs méthodes basées sur la minimisation d'un critère existent. Quelque soit la méthode retenue, il y a une première question importante mais souvent négligée à se poser : « est-il possible d'identifier les paramètres désirés à partir des données disponibles? » Cette première question peut être reliée à la notion de "sensibilité ». Elle dépend du lien reliant les paramètres aux sorties, mais aussi des précisions souhaitées sur ces paramètres et de la précision disponible sur les mesures. Le concept d'équifinalité souligne le fait que, dans certains cas, plusieurs jeux de paramètres peuvent fournir les mêmes sorties d'un modèle, modulo des incertitudes données. Ce concept est sujet à caution, car il est utilisé pour critiquer un modèle, intrinsèquement, alors qu'il est tout autant lié au jeu de données utilisé pour le calage. Une question importante est donc « quel serait le jeu de données optimal pour le calage ? » Le concept de "pire cas » peut apporter des éléments de réponse à cette question, sous certaines hypothèses. L'article présente des méthodologies permettant de répondre partiellement à ces questions, et les illustre sur un exemple de la littérature. Cela montre qu'un algorithme de minimisation peut être utile, mais que savoir répondre aux deux questions posées ci-dessus l'est tout autant.
\end{abstract}

Mots clefs : Sensibilité, Diagramme de Bode, Norme $\ell_{1}$, Perturbation pire-cas, Scenario d'entrée

\section{Analysis for the calibration of open-channel flow models : insights from linear systems and automatic control approaches}

\begin{abstract}
Any project using a model should include a calibration phase. For a 1D hydrodynamic model based on Saint-Venant's equations, this often means adjusting roughness coefficients, discharge coefficients at cross and lateral devices, and seepage. Several methods are described in the literature, based on minimization approaches. Whatever algorithm selected, there is nevertheless an important question to address first, which is seldom studied in the literature : « is it possible to identify the parameters I want from the measurements I have ? » This first question is linked to what is called «sensitivity». This depends on the relationship between parameters and outputs of the model, but also on the desired precision on the parameters and on the available one on the measurements. Some people introduced the concept of « equifinality» stating that, in some cases, several different sets of parameters give the same model outputs within given uncertainties. This concept is somehow peculiar in the sense that it is used to criticize the model itself, whereas it may also be attached to the set of data used for the calibration. An important question is therefore "what type of input scenario would be best to be able to calibrate the model ? » This second question can be addressed under some assumptions using the «worst-case» concept. The paper provides methodologies for partially answering these 2 questions, and is applied to an example taken from the literature. This shows that having a minimization algorithm is maybe useful, but being able to answer the two above questions is even more important.
\end{abstract}

Key words : Sensitivity, Bode plot, $\ell_{1}$ norm, Worst-case perturbation, Input scenario

\section{INTRODUCTION}

Le calage d'un modèle hydraulique [1] est une question qui se pose pour tout projet utilisant un tel outil. On pourrait discuter de la façon dont ce calage doit être fait, afin d'être confiant sur la validité des conclusions tirées de l'utilisation du modèle.

Lorsque des modèles hydrauliques sont utilisés pour la conception de contrôleurs, pour l'automatisation d'ouvrages, leur calage est une question importante pour plusieurs rai- sons. La dynamique transitoire doit être correctement représentée sur une plage de fréquences couvrant le domaine de sollicitation du système. Les méthodologies de conception de contrôleur doivent vérifier a posteriori, ou imposer $a$ priori, les conditions de robustesse qui permettent certains écarts du modèle utilisé pour leur conception. Néanmoins, évaluer ces incertitudes de modèle et essayer de les réduire est toujours important, quoique rarement fait en pratique.

Nous sommes d'accord avec les avertissements donnés par des experts en hydraulique sur un calage « automatique » 
des modèles [3]. Mais, en même temps, le développement d'outils de calage « intelligents » peut être utile pour plusieurs raisons [4].

La question est alors : «comment caler un modèle ?» Pour un modèle hydrodynamique 1D basé sur les équations de Saint-Venant [1], cela signifie souvent ajuster les coefficients de rugosité de Manning-Strickler, les coefficients de débit des ouvrages, et les valeurs d'infiltration. La littérature propose plusieurs méthodes, basées sur des techniques de minimisation ([5], [6], [7], [8]). Des travaux récents utilisent des approches de type Monte Carlo ou le filtrage de Kalman pour reconstruire des entrées inconnues [9] et des coefficients de Strickler [10]. Certaines de ces méthodes sont programmées dans des logiciels hydrauliques tels que le logiciel SIC (Cemagref) ou Dassflow [8].

Quel que soit l'algorithme choisi pour ce calage, il y a néanmoins une question importante à traiter en premier : « est-il possible d'identifier les paramètres que je veux à partir des mesures que j'ai ? » Cette première question est liée à ce que l'on appelle la «sensibilité » [11]. Cela dépend de la relation entre les paramètres et les sorties du modèle, mais aussi de la précision souhaitée sur les paramètres et sur celle disponible sur les mesures. Les automaticiens ont également définis le concept $\mathrm{d}$ « observabilité » conduisant à un critère algébrique indiquant si les états internes du modèle peuvent être reconstruits à partir de la mesure des sorties pendant une certaine durée. Ce concept est assez générique, car les états internes peuvent inclure des paramètres et des entrées inconnues [2], [9], [10].

Certaines personnes ont introduit le concept d' «équifinalité » [12] en indiquant que, dans certains cas, plusieurs ensembles de paramètres donnent les mêmes sorties du modèle (à une précision près). Ce concept est à clarifier dans le sens où il est parfois utilisé pour critiquer le modèle lui-même (et ses excès de paramètres), alors qu'il dépend également des jeux de données utilisés pour son calage ou sa validation. Une question importante est donc «quel scénario d'entrée serait préférable pour caler le modèle ? » Une autre question, si le scénario d'entrée est imposé, est «les caractéristiques de mon scénario sont-elles assez riches pour caler certains ou tous les paramètres de mon modèle ? " Après une présentation du cadre général du calage, nous présenterons des méthodes permettant de répondre en partie à ces questions. Des illustrations seront données sur un exemple tiré de la littérature [6].

\section{APPROCHES DU CALAGE}

\section{II.1. Position du problème}

Un modèle hydraulique peut être considéré, d'un point de vue mathématique, comme un opérateur $\Phi$ :

$$
\begin{gathered}
\Phi: E^{n} \rightarrow E^{m} \\
u \mapsto y=\Phi(u) \\
\operatorname{avec} y(t)=f(u(t), x(t), p, t)
\end{gathered}
$$

Où $E^{1}$ est un espace dimension $l$ (habituellement $\ell_{\infty}^{l}\left(Z_{+}\right)$, espace des séries à valeurs vectorielles réelles bornées définies sur les entiers positifs - représentant les instants temporels - , de dimension $l$ ), $u$ est le vecteur (dimension $n$ ) des entrées du modèle (e.g. : débits aux nœuds amont, cotes aux nœuds aval, positions des vannes ou seuils intermé- diaires), et $y$ est le vecteur (dimension $m$ ) des sorties du modèle (e.g. : cotes et débits aux mêmes endroits). Cet opérateur est, dans le cas général, défini au travers d'une fonction $f$ de diverses variables et de paramètres tels que : $x$ un vecteur des états internes (e.g. : cotes et débits aux sections de calcul, distantes d'un pas d'espace $\Delta x), p$ un vecteur de paramètres, et $t$ le temps (habituellement des valeurs échantillonnées au pas de temps $\Delta t$ ). Les variables $y, u$ et $x$ sont des fonctions du temps $t$. Le vecteur des paramètres $p$ est supposé constant au cours du temps. La fonction $f$ est, dans notre cas, une fonction non-linéaire obtenue sous forme numérique après discrétisation des équations de Saint-Venant par le schéma implicite de Preissmann [13]. D'autres schémas sont disponibles et ce qui suit reste valide.

Le vecteur des paramètres $p$ est habituellement composé de différentes choses :

$p_{\mathrm{n}}$ : les paramètres numériques (e.g. : coefficient d'implicitation du schéma numérique, coefficient de relaxation d'un algorithme de Newton-Raphson, test de convergence d'un algorithme itératif, etc.),

$p_{\mathrm{g}}$ : les paramètres géométriques (e.g. : sections en travers, dimensions des vannes ou des seuils),

$p_{\mathrm{h}}$ : les paramètres hydrauliques (e.g. : coefficients de frottement à chaque section de calcul, les coefficients de débit des vannes ou des seuils).

Si les mesures des sorties $y_{\mathrm{m}}$ sont disponibles à divers instants sur un horizon $\left[T_{1}, T_{2}\right]$ (incluant le cas particulier $T_{1}=T_{2}$ ), il est alors possible d'essayer de caler le modèle. Sans perte de généralité on peut faire l'hypothèse que toutes les sorties $y$ sont mesurées, car la fonction $f$ peut être ajustée si nécessaire pour remplir cette condition. L'étape de calage consiste alors à trouver un vecteur de paramètres $p$ minimisant le critère d'erreur sur les sorties du modèle :

$$
p=\operatorname{Arg} \min J\left(y, y_{m}\right),
$$

où $J$ est un critère défini à partir du vecteur de sortie $y$ comme défini dans (1) et du vecteur de mesure $y_{\mathrm{m}}$ correspondant. Nous pouvons, par example, choisir un critère quadratique classique :

$$
J=\sum_{t \in\left[T_{1}, T_{2}\right]}\left\|y-y_{m}\right\|^{2}
$$

\section{II.2. Algorithmes}

\section{II.2.1. Régime permanent}

Plusieurs algorithmes de calage ont été développés par divers auteurs, et décrits dans les documentations des logiciels ou dans des articles. Pour les régimes permanents, une procédure itérative basée sur la méthode du gradient a été mise en œuvre dans le logiciel SIC et donne des résultats satisfaisants [5].

Le calage en régime permanent présente plusieurs avantages, bien entendu dans les cas où de telles conditions peuvent être obtenues. Les mesures peuvent être prises par quelques personnes se déplaçant le long du système, en quelques minutes, heures ou jours, selon la taille du système. L'algorithme utilisé peut être assez simple, surtout si un seul coefficient de rugosité doit être calé pour chaque partie du système ayant un jeu de mesures $\left(y_{\mathrm{m} \text { up }}, y_{\mathrm{m} \text { down }}, Q_{\mathrm{up}}\right)$. D'autres algorithmes sont disponibles pour des situations plus complexes, mais nous pouvons atteindre la limite de l'observabilité de la même manière que pour les écoule- 
ments transitoires étudiés dans les sections suivantes. Le calage en régime permanent a également de nombreuses limites. Tout d'abord, il est souvent difficile d'avoir de véritables conditions d'écoulement permanentes sur des rivières naturelles de grande taille ou sur des canaux d'irrigation. Les rivières naturelles ont des entrées qui ne peuvent pas être contrôlées explicitement, et il est difficile de demander à un gestionnaire de canal d'irrigation de ne pas bouger ses ouvrages pendant plusieurs jours. En outre, les procédures de calage correspondantes n'exploitent pas les informations dynamiques potentiellement intéressantes afin d'obtenir un bon modèle hydrodynamique (par exemple : temps de retard, modes d'oscillation). En conséquence, même si nous pouvons très bien calibrer un modèle en régime permanent, nous n'avons aucune garantie que le modèle sera également une bonne représentation transitoire du système réel. Une procédure classique est donc de caler un modèle pour un régime permanent, et de vérifier si le modèle reste satisfaisant pour une ou plusieurs conditions d'écoulement transitoires. Dans le cas où il ne l'est pas, que faire ? Le calage en régime transitoire peut être une option!

\section{II.2.2. Régime transitoire}

Plusieurs auteurs ont suggéré et testé des algorithmes pour résoudre ce problème de calage en régime transitoire [8]. Dans les chapitres suivants nous allons utiliser l'exemple décrit dans [6]. Dans cet article, les auteurs callent les coefficients de rugosité de Manning pour un bief de rivière fictif de $20 \mathrm{~km}$ de long, comprenant 7 sections de calcul, en utilisant des mesures des niveaux d'eau en amont et en aval. L'entrée du modèle est le débit en amont. Le bief a une pente longitudinale de 0,002. Le coefficient de Manning augmente linéairement de 0,02 pour $X=0$ à 0,03 pour $X=20 \mathrm{~km}$. La section est trapézoïdale, avec un fruit de 4 , et la largeur au fond est de $30 \mathrm{~m}$. Le débit nominal n'est pas spécifié (nous avons pris $\left.200 \mathrm{~m}^{3} / \mathrm{s}\right)$. La condition limite aval n'est pas spécifiée (nous avons pris une loi uniforme correspondant aux données de la section aval). Le scénario d'entrée, réalisé dans le cadre d'une expérience jumelle, n'est pas spécifié, sauf que sa durée est de 50 minutes. L'algorithme utilisé est basé sur une méthode d'optimisation avec multiplicateurs de Lagrange, et descente du gradient. Les auteurs indiquent qu'ils retrouvent, après 21 itérations, les bons coefficients de Mannings avec une erreur faible.

\section{ANALYSE DE SENSIBILITÉ ET SCENARIO D'ENTRÉE}

Lorsqu'une méthode de calage prétend pouvoir caller $n$ paramètres $p_{1}, \ldots$, n (e.g. : dans notre cas $n=7$ ) simultanément, à partir d'un jeu de données (e.g. : dans notre cas $\left(y_{\mathrm{m} \text { up }}, y_{\mathrm{m} \mathrm{down}}, Q_{\mathrm{up}}\right)_{[\mathrm{T} 1}$ T T2 $]$ avec $\left.T_{1}=0, T_{2}=50 \mathrm{mn}\right)$, elle devrait également être capable de caler un seul d'entre eux $\left(p_{\mathrm{i}}\right)$, si les $n-1$ autres sont supposés déjà connus. Avant de lancer un programme de calage, on devrait au préalable vérifier que les sorties du modèle $\left(y_{\text {up }}, y_{\text {down }}\right)$ sont sensibles à ce paramètre $p_{\mathrm{i}}$ [14]. Une méthode classique et simple pour faire cela consiste à réaliser 2 simulations avec 2 valeurs différentes pour $p_{\mathrm{i}}\left(p_{\mathrm{i}}\right.$ et $\left.p_{\mathrm{i}}+\Delta p_{\mathrm{i}}\right)$ et regarder si les sorties du modèle sont différentes. La méthode GLUE est basée sur ce principe, en utilisant une approche de type Monte Carlo avec des paramètres choisis aléatoirement dans l'intervalle $\left[p_{\mathrm{i}}, p_{\mathrm{i}}+\Delta p_{\mathrm{i}}\right]$. Dans le cas de modèles à beaucoup de paramètres, elle peut conduire à des millions of simulations [12]. D'autres méthodes basées sur des approches variationnelles faisant appel au modèle adjoint [8], [15] ou sur l'intégration numérique du modèle de sensibilité [16] permettent de faire cette analyse de manière plus efficace. Toutes ces méthodes font cependant cette analyse pour un choix particulier de scénario d'entrée (ou le reproduise pour un petit nombre de scénarios). Ce n'est pas le cas de la méthode que nous proposons dans cet article.

Nous savons que, dans la plupart des cas (sauf si le bief est assez long pour que l'écoulement uniforme soit atteint, ou si la profondeur critique est atteinte, etc.), cette sensibilité existe, et le problème du calage de $p_{\mathrm{i}}$ sera facile à résoudre, car il peut même été résolu avec un simple jeu de données en régime permanent.

Nous pouvons penser à un cas un peu plus complexe, lorsque deux paramètres $\left(p_{\mathrm{i}}\right.$ and $\left.p_{\mathrm{j}}\right)$ sont inconnus et doivent donc être calés simultanément. Dans ce cas, un seul jeu de données en régime permanent ne peut pas permettre de résoudre le problème (deux inconnues pour une seule équation), et il existe un nombre infini de solutions (ou pas de solution). Dans notre exemple issu de [6], les coefficients de Strickler aux sections 4 et 6 avec des valeurs nominales $(\mathrm{K}(4)=40, \mathrm{~K}(6)=35.2939)$ et des valeurs alternatives $(\mathrm{K}(4)=31.62891, \mathrm{~K}(6)=65.2939)$ donnent exactement les mêmes valeurs de sortie $\left(y_{\text {up }}, y_{\text {down }}\right)$ pour un débit d'entrée $\mathrm{Q}=200 \mathrm{~m}^{3} / \mathrm{s}$ à une précision supérieur à $10^{-5} \mathrm{~m}$. Dans ce cas, nous pouvons penser à résoudre le problème avec un jeu de données en régime transitoire (une autre option aurait été d'utiliser plusieurs jeux de données en régime permanent). Les questions suivantes peuvent alors être posées :

Est-il possible de retrouver à partir du jeu de données mesurées $\left(y_{\mathrm{m} \text { un }}, y_{\mathrm{m} \text { down }}\right)_{[\mathrm{T} 1}$ T $\left.\mathrm{T} 2\right]$ la combinaison nominale correcte de $\mathrm{K}(4)$ et $\mathrm{K}(6)$ ? Est-il possible de quantifier la facilité avec laquelle cette détection peut se faire (par exemple dans le cas où nous voulons tenir compte de la précision finie nous avons sur les mesures)? Quel est le meilleur scenario $\left(Q_{\mathrm{up}}\right)_{\left[\mathrm{T} 1, \mathrm{~T}_{2}\right]}$ qui maximise la sensibilité de $\left(p_{\mathrm{i}}, p_{\mathrm{j}}\right)$ sur les mesures réalisées?

Des méthodes d'analyse de sensibilité globales avec plusieurs paramètres existent (e.g. : Hypercubes Latin, Morris, Sobol, Metamodèles, etc.). Mais, pour les modèles transitoires, le choix du scenario d'entrée est très important et ne peut pas être facilement ajouté dans une analyse de sensibilité classique (du fait du nombre important des nouvelles variables qu'il faudrait ajouter). Puisque nous voulons focaliser notre analyse sur l'influence du scenario d'entrée (sans oublier la sensibilité sur les paramètres $p$ ) nous allons proposer ici d'étudier l'opérateur différence $\Phi_{\mathrm{d}-\mathrm{nl}}$ obtenu comme la différence entre les deux opérateurs $\Phi_{1-n l}$ et $\Phi_{2-n l}$ : $\Phi_{\mathrm{d}-\mathrm{nl}}=\Phi_{2-\mathrm{nl}}-\Phi_{1-\mathrm{n} \text {, }}$, pour une différence donnée $\Delta p$ sur le vecteur des paramètres $p$, avec de manière plus précise :

$$
\begin{aligned}
\Phi_{1-n l}: u(t) & \mapsto y_{1}(t)=f\left(u(t), x_{1}(t), p, t\right) \\
\Phi_{2-n l}: u(t) & \mapsto y_{2}(t)=f\left(u(t), x_{2}(t), p+\Delta p, t\right)
\end{aligned}
$$

Pour être en mesure d'utiliser les puissants outils de l'analyse des systèmes, nous approximons ces opérateurs par leur représentation linéaire dans l'état-espace et en temps discret :

$$
\begin{gathered}
\Phi_{1}:\left\{\begin{array}{c}
x_{1}^{+}=A_{1} x_{1}+B_{1}\left(u-u_{1_{0}}\right) \\
y_{1}=y_{1_{0}}+C_{1} x_{1}+D_{1}\left(u-u_{1_{0}}\right)
\end{array}\right. \\
\Phi_{2}:\left\{\begin{array}{c}
x_{2}^{+}=A_{2} x_{2}+B_{2}\left(u-u_{2_{0}}\right) \\
y_{2}=y_{2_{0}}+C_{2} x_{2}+D_{2}\left(u-u_{2_{0}}\right)
\end{array}\right.
\end{gathered}
$$


avec $u_{\mathrm{i} 0}$ le vecteur d'entrée initial de référence au temps $0, y_{\mathrm{i} 0}$ le vecteur de sortie initial correspondant pour les operateurs $\Phi_{\mathrm{i}}(\mathrm{i}=1,2)$. Dans notre cas simple décrit ci-dessus nous avons $u_{10}=u_{20}$ et $y_{10}=y_{20} . \mathrm{A}_{\mathrm{i}}, \mathrm{B}_{\mathrm{i}}, \mathrm{C}_{\mathrm{i}}$ et $\mathrm{D}_{\mathrm{i}}$ sont des matrices de dimensions appropriées et dépendent (non-linéairement) $\mathrm{du}$ vecteur des paramètres $p$. Ces matrices sont obtenues à partir de la linéarisation de la fonction $f$ (Equation 1) autour d'un état nominal $x_{\mathrm{i} 0}$. A partir d'ici, et dans les chapitres suivants, nous supposons que les matrices $A_{i}, B_{i}, C_{i}$ et $D_{i}$ sont constantes dans le temps (nous nous plaçons dans le cadre appelé LTI pour « Linear Time Invariant»). Les effets non-linéaires ont été étudiés et bien que ne pouvant pas être détaillés dans cet article faute de place seront évoqués rapidement en fin d'article.

Nous pouvons maintenant étudier la sensibilité du vecteur de paramètres $p$ via l'étude de l'opérateur $\Phi_{\mathrm{d}}$.

\section{ANALYSE FREQUENTIELLE (DIAGRAMME DE BODE)}

Nous pouvons étudier les caractéristiques fréquentielles de l'opérateur $\Phi_{d}$ par le diagramme de Bode (Figure 1). Ce graphique montre l'amplitude et la phase de la sortie en fonction de la fréquence d'une entrée sinusoïdale d'amplitude 1 $\left(\mathrm{m}^{3} / \mathrm{s}\right.$ dans notre cas, puisque l'entrée est le débit en amont).

De l'analyse qui précède nous pouvons dire qu'afin de caler simultanément les coefficients de Stricklers aux sections 4 et 6 , pour un scénario d'entrée de type sinusoïdal, il serait optimal de sélectionner une fréquence autour de $\mathrm{F}_{\text {opt up }}=1.1110-3 \mathrm{rad} / \mathrm{s}$ (correspondant à une période de $5646 \mathrm{~s}$ ) dans le cas où la cote de l'eau à l'amont du bief est utilisée pour ce calage, et $\mathrm{F}_{\text {opt down }}=1.0010-3 \mathrm{rad} / \mathrm{s}$ (correspondant à une période de $6283 \mathrm{~s}$ ) pour l'utilisation de la cote de l'eau à l'aval. Puisque nous utilisons deux mesures de niveau d'eau (amont et aval), nous avons intérêt à utiliser un scénario d'entrée avec une énergie suffisante dans ces deux fréquences. Nous avons vérifié sur quelques essais que ces fréquences sont principalement liées à la position des Stricklers 4 et 6 , mais pas à l'amplitude $\Delta p$ sélectionnée.
Ce point devra être étudié plus en détail et sera intégré dans un prochain article. Nous pouvons également observer dans la Figure 1 et Table 1 que le gain maximal $(-63.62 \mathrm{~dB})$ est obtenu pour la mesure de niveau d'eau à l'aval à la fréquence $\mathrm{F}_{\text {opt down }}$. Comme dit précédemment, nous ne connaissons pas précisément le scénario retenu par les auteurs de l'article [6]. Mais, du fait qu'il dure $3000 \mathrm{~s}$, on peut imaginer que sa fréquence de base peut être de $\pi /(2 * 3000)=5.2410^{-4}(\mathrm{rad} / \mathrm{s})$ (pour un quart de cycle de sinusoïde) à $2 \pi / 3000=2.0910^{-3}$ $(\mathrm{rad} / \mathrm{s})$ (pour un cycle complet de sinusoïde). Si les auteurs ont sélectionné ce scénario d'entrée par hasard, ils ont eu de la chance car ces fréquences sont proches de celles optimales mises en évidence ci-dessus. Une autre question peut être : quelle doit être l'amplitude du scénario d'entrée ? Un gain de $-63.62 \mathrm{~dB}$ correspond à $\Delta \mathrm{Z}=10^{-63.62 / 20}=0.66 \mathrm{~mm}$ d'écart maximum (du niveau d'eau en aval) entre les 2 modèles pour un scénario d'entrée optimal (sinusoïdal d'amplitude $1 \mathrm{~m}^{3} / \mathrm{s}$ ) à la fréquence $\mathrm{F}_{\text {opt down }}$. Si la précision de mesure est de $1 \mathrm{~cm}$, l'amplitude du scénario d'entrée doit donc être d'au moins $15 \mathrm{~m}^{3} / \mathrm{s}(0.01 / 0.00066)$ pour être en mesure de détecter une différence entre $\Phi_{1}$ et $\Phi_{2}$. Ceci est correct si les effets non-linéaires négligés n'introduisent pas un amortissement supplémentaire, ce qui a été vérifié. Avec un amortissement non-linéaire supplémentaire, cette amplitude devra être encore augmentée. Avec d'autres choix sur le scénario d'entrée, nous pouvons affirmer que ce ne sera pas possible de caler les Stricklers simultanément aux sections 4 et 6 avec une précision meilleure que l'écart $\Delta p$ que nous avons introduit sur les paramètres $(\Delta \mathrm{K}(4)=8.4, \Delta \mathrm{K}(6)=30)$.

\section{ANALYSIS TEMPORELLE (NORME $\ell_{1}$ )}

\section{V.1. Calcul de la norme $\ell_{1}$}

Les développements réalisés ci-dessus à partir des diagrammes de Bode sont basés sur des scénarios choisis dans l'espace des fonctions sinusoïdales. On peut se demander quels seraient les écarts maximums sur les sorties de l'opérateur $\Phi_{\mathrm{d}}$ pour n'importe quel scenario d'entrée, sans
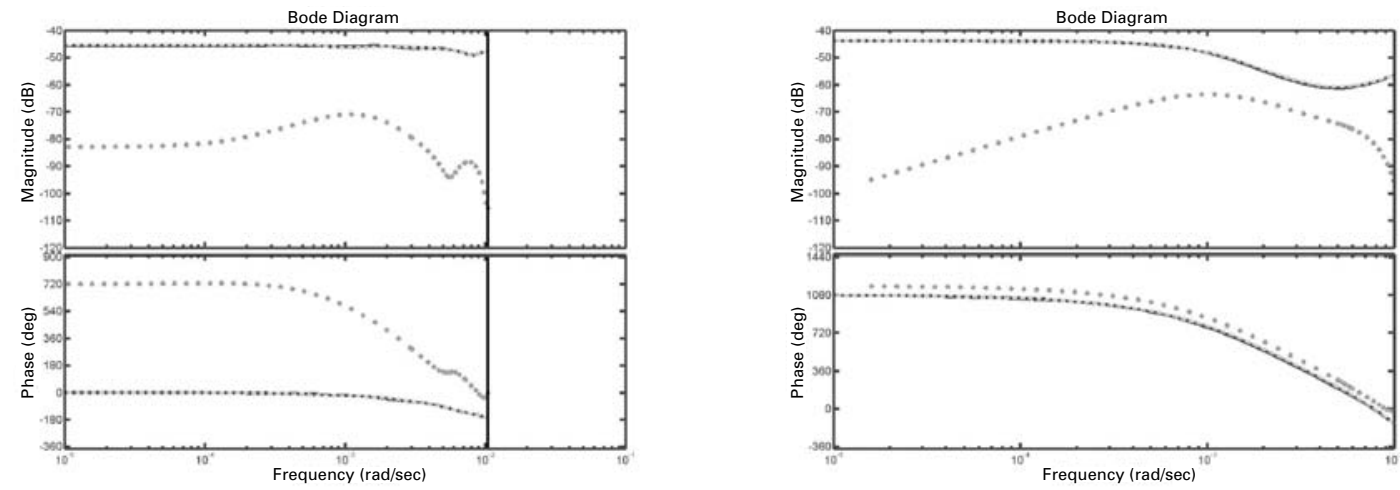

Figure 1 : Diagramme de Bode des opérateurs $\Phi_{1}$ (points rouge proches de la ligne jaune), $\Phi_{2}$ (ligne jaune proche des points rouges) et $\Phi_{d}$ (points verts plus éloignés des courbes rouge et jaune) pour les sorties correspondants à la cote amont (figure de gauche) et cote aval (figure de droite).

Table 1: Valeurs clefs du diagramme de Bode.

\begin{tabular}{|l|c|c|c|c|c|c|}
\hline Emplacement & $\mathbf{\Delta} \mathbf{Z}_{\text {static }}(\mathbf{d B})$ & $\boldsymbol{\Delta} \mathbf{Z}_{\text {static }}(\mathbf{m m})$ & $\boldsymbol{\Delta} \mathbf{Z}_{\max }(\mathbf{d B})$ & $\boldsymbol{\Delta} \mathbf{Z}_{\max }(\mathbf{m m})$ & Freq. pour $\boldsymbol{\Delta} \mathbf{Z}_{\max }(\mathbf{r a d} / \mathbf{s})$ & $\mathbf{T s}$ pour $\Delta \mathbf{Z}_{\max }(\mathbf{s})$ \\
\hline Amont & -82.91 & 0.07 & -71.02 & 0.28 & $1.1110^{-3}$ & 5646 \\
\hline Aval & $-\infty$ & 0 & -63.62 & 0.66 & $1.0010^{-3}$ & 6283 \\
\hline
\end{tabular}


contrainte sur sa forme ni sa fréquence (sa seule contrainte étant qu'il doit être borné, ce qui est clairement réaliste). Après normalisation de l'opérateur $\Phi_{d}$ nous pouvons écrire cette contrainte sous la forme $\|u\|_{\ell_{\infty}} \leq 1$. Si nous recherchons l'écart maximum des sorties $\|y\|_{\ell_{\infty}}$ sous la contrainte précédente, nous recherchons par définition la norme induite $\ell_{\infty}-\ell_{\infty}$ de l'opérateur $\Phi_{\mathrm{d}}$. Il peut être démontré facilement que cette norme induite est la norme $\ell_{1}$ [2].

$$
\left\|\Phi_{d}\right\|_{\ell_{1}}=\left\|\Phi_{d}\right\|_{\text {ind } \ell_{\infty}-\ell_{\infty}}=\sup _{|u|_{\ell_{\infty}} \leq 1}\|y\|_{\ell_{\infty}}
$$

Dans la Figure 2 nous représentons cette norme pour chaque section en travers $(\mathrm{n}=1$ à 7$)$ le long du système, en terme de débit et de cote.

Nous pouvons vérifier que la sensibilité des paramètres $(\mathrm{K}(4), \mathrm{K}(6))$ est plus forte vers les niveaux à l'aval qu'à l'amont. Nous pouvons également observer que la sensibilité la plus forte est obtenue à la section $n^{\circ} 6$. Nous remarquons enfin qu'il existe un scenario pire-cas au sens de la norme $\ell_{1}$ avec une sensibilité plus forte que le pire-cas sinusoïdal, puisque la norme est $1.28 \mathrm{~mm}$ comparée aux $0.66 \mathrm{~mm}$ pour $\Delta \mathrm{Z}_{\mathrm{dn}}$ (et $0.42 \mathrm{~mm}$ comparée aux $0.28 \mathrm{~mm}$ pour $\Delta \mathrm{Z}_{\mathrm{up}}$ ).

\section{V.2. Calcul du scénario pire-cas}

Un aspect intéressant de la norme $\ell_{1}$ est qu'il est facile de calculer ses scénarios pire-cas [2], et même de manière plus réaliste ses scénarios e-pire-cas. Lorsque nous les calculons pour l'opérateur $\Phi_{\mathrm{d}}$, cela fourni le scénario d'entrée $u=\left(Q_{\text {up }}\right)_{[T 1},{ }_{\text {T2 }]}$ (avec norme $\left.\ell_{\infty}=1\right)$ donnant la sortie maximum $y=\left(y_{\text {up }}, y_{\text {down }}\right)_{[T 1, T 2]}$ (au sens de sa norme $\left.\ell_{\infty}\right)$. Du fait de la définition de $\Phi_{\mathrm{d}}=\Phi_{2}-\Phi_{1}$ cela donne le meilleur scenario d'entrée permettant de discriminer entre les opérateurs $\Phi_{1}$ et $\Phi_{2}$, i.e. permettant de détecter le vecteur d'écart sur les paramètres $\Delta p$ que nous avons introduit, à partir du jeu de

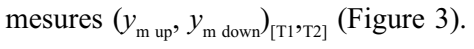

\section{CONCLUSIONS}

Cet article ne présente pas un nouvel algorithme pour caler les coefficients de rugosité, ni en régime permanent, ni en régime transitoire. Ce document présente par contre des éléments de méthodologie pour l'analyse d'un système à caler, basés sur des outils issus des théories de l'analyse des systèmes linéaires et de l'automatique. Deux différentes approches complémentaires ont été présentées : une basée
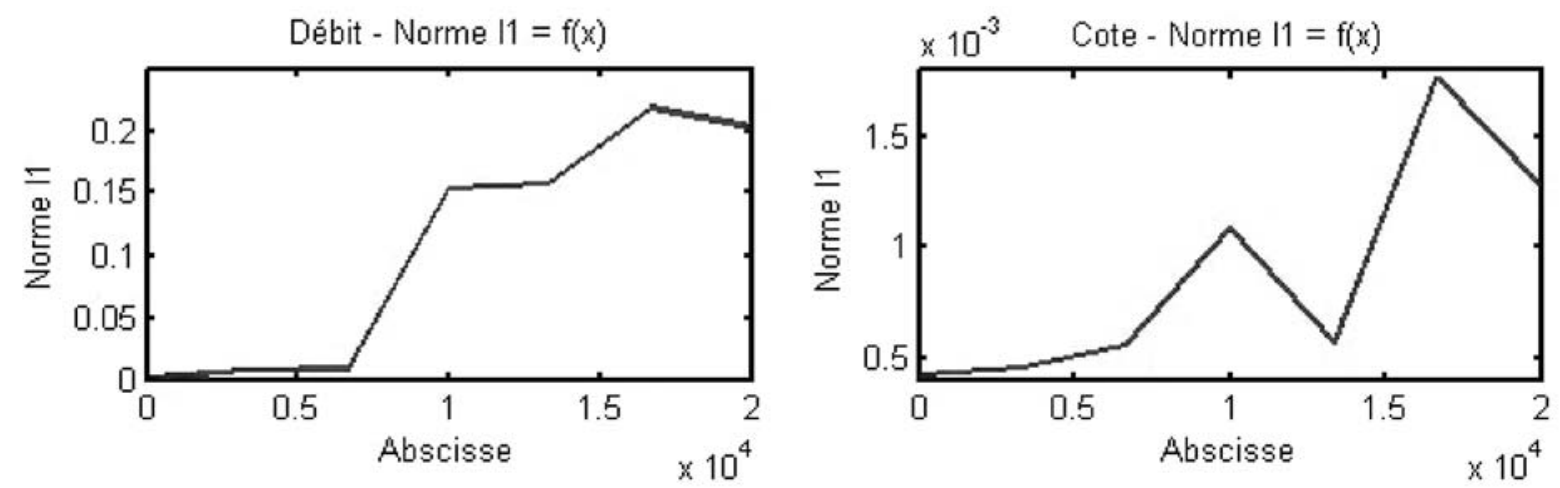

Figure 2 : Norme $\ell_{1}$ de l'opérateur différence $\Phi_{d}$ le long du système. Cette norme est calculée pour les sorties correspondant aux débits (figure de gauche) et aux cotes (figure de droite).

Table 2: Valeurs clef de l'analyse par la norme $\ell_{1}$

\begin{tabular}{|l|c|c|}
\hline \multicolumn{1}{|c|}{ Emplacement } & norme $\ell_{1} \operatorname{sur} \Delta \mathbf{Q}\left(\mathbf{m}^{3} / \mathbf{s}\right)$ & norme $\ell_{1} \operatorname{sur} \Delta \mathbf{Z}(\mathbf{m m})$ \\
\hline Amont & 0 & 0.42 \\
\hline Aval & 0.202 & 1.28 \\
\hline
\end{tabular}
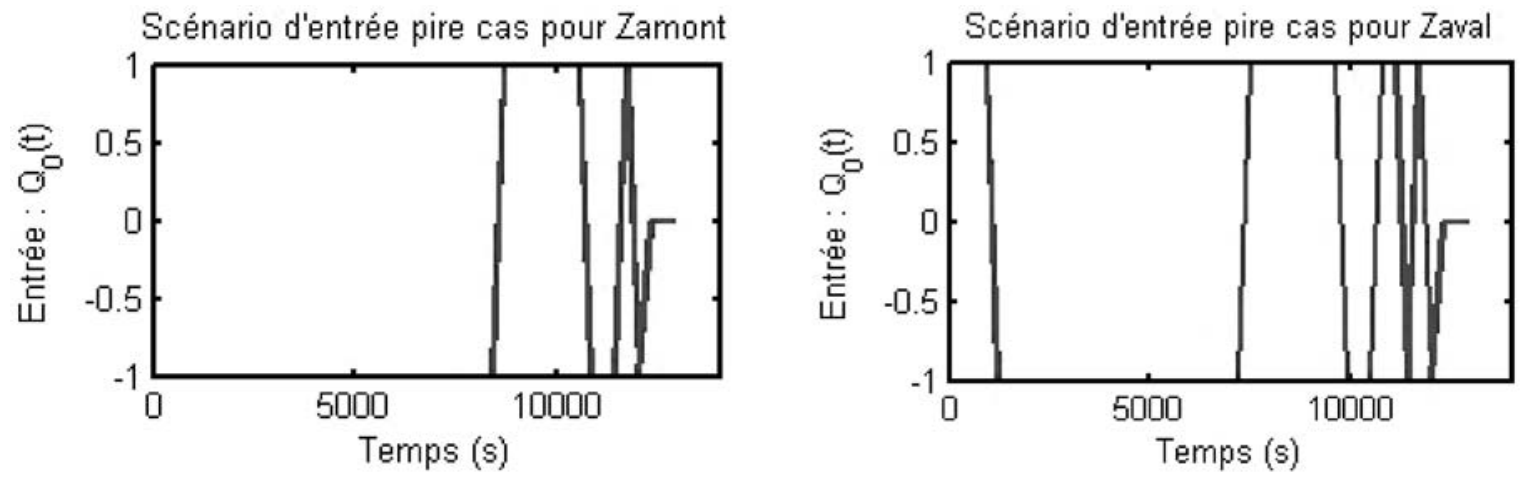

Figure 3 : Scénario pire-cas d'entrée $Q_{0}(t)$ pour la norme $\ell_{1}$ pour l'opérateur différence $\Phi_{d^{\prime}}$ Ce scenario est calculé pour les sorties correspondant aux cotes amont (figure de gauche) et cotes aval (figure de droite). 
sur une analyse fréquentielle à partir des diagrammes de Bode, et l'autre sur une analyse temporelle à partir du calcul d'une norme $\ell_{1}$. La première peut donner des indications sur la sensibilité de certains paramètres du modèle, sur l'ampleur et la fréquence optimale d'un scénario d'entrée sinusoïdal qui peut être choisi pour le calage des paramètres du modèle. La seconde peut donner des indications supplémentaires, telles que le meilleur emplacement pour les mesures utilisées pour le calage (cela peut également être fait avec la méthode fréquentielle précédente quitte à calculer le diagramme de Bode pour chaque section), l'ampleur d'un scénario d'entrée qui peut être sélectionné pour le calage, ou le scénario "pire-cas » qui peut être plus complexe qu'une sinusoïde avec un meilleur effet discriminant pour une valeur maximale d'entrée identique.

Ces outils peuvent être vus comme un moyen de quantifier le concept équifinalité en faisant apparaitre un lien qui existe entre les scénarios d'entrée du modèle et ses sorties. En outre, cette dernière méthode est générique pour systèmes multivariables, au cas où le scénario d'entrée peut être appliqué sur plusieurs entrées u (par exemple : le débit en amont, les apports latéraux et/ou les positions des ouvrages situés le long du système). Dans le cas où le scénario d'entrée ne peut pas être imposé de manière libre sur un système réel, la méthodologie peut également permettre d'évaluer la qualité du scénario d'entrée imposé, à partir d'une analyse en fréquence et en amplitude par décomposition de Fourier dans ses modes principaux. De nombreuses applications de cette méthodologie peuvent être prévues au-delà de l'exemple simple utilisé dans le présent article : l'évaluation de l'influence des paramètres numériques des schémas numériques, l'importance des différents termes des équations de SaintVenant (par exemple les termes d'inertie), le comportement dynamique en fonction de l'état nominal, etc.

Les deux principales contraintes imposées par la méthode sont (i) qu'elle est basée sur le modèle linéaire tangent, et (ii) que la partie dynamique est la seule analysée. Nous avons vérifié que dans notre cas les non-linéarités ne changeaient pas sensiblement les résultats, puisqu'avec des amplitudes des scénarios d'entrées multipliées jusqu'à des facteurs 150 , les écarts relatifs sur les opérateurs différences étaient inférieurs à $10 \%$. En ce qui concerne la partie statique, elle est en général plus simple à analyser, et elle est indépendante de certains paramètres (ceux du schéma numérique par exemple, ou dans le cas de compensation de certains paramètres comme dans l'exemple que nous avons choisi).

L'apport important de l'approche présentée ici par rapport à toutes les autres méthodes décrites dans la littérature, y compris les approches basées sur les méthodes variationnelles ou sur l'intégration du modèle de sensibilité, est que cette analyse est faite pour un ensemble infini de scénarios d'entrée (scénarios sinusoïdaux de fréquences définies dans un intervalle de fréquence, ou ensemble des scénarios bornés dans le cadre de l'utilisation de la norme $\ell_{1}$ ). Il est certain que la généralisation de cette approche aux cas fortement non-linéaires serait très intéressante, mais a priori avec des outils mathématiques à découvrir !

\section{RÉFÉRENCES ET CITATIONS}

[1] Cunge J. A., F. M. Holly, Et Al. (1980) — Practical aspects of computational river hydraulics. Pitman Advanced Publishing Program
[2] Dahleh M. A., \& Diaz-Bobillo I. J. (1995) - Control of uncertain systems : a linear programming approach. PrenticeHall

[3] Cunge J. (2003) - Of data and models. Journal of Hydroinformatics. 5(2) 75-98

[4] Fread D. L., \& SMith G. F. (1978) - Calibration technique for 1-D unsteady flow models. Journal of the Hydraulics Division. Proceedings of the ASCE. 104(HY7) 1027-1044

[5] Pouchelle D. (1991) - Détermination Algébrique des Coefficients de Strickler. Cemagref, Ecole Nationale Supérieure d'Hydraulique et de Mécanique Grenoble

[6] Atanov G., Evseeva E., \& Meselhe, E (1999) - Estimation of roughness profile in trapezoidal open channels. J Hydraul Eng. 125(3) 309-311

[7] Das A. (2004) - Parameter estimation for flow in open-channel networks. Journal of Irrigation and Drainage Engineering. 130(2) 160-165

[8] Honnorat M., Marin J., Monnier J., \& Lai X. (2007) Dassflow 1.0 : a variational data assimilation software for $2 D$ River flows. INRIA

[9] Malaterre P.-O., Jean-Baptiste N., Doree C., \& Sau J. (2009) - Data assimilation for real-time estimation of hydraulic states and unmeasured perturbations in a 1D hydrodynamic model. Application to water management problems and comparison of Kalman filter and sequential Monte Carlo approaches. MAMERN09, 3rd Mamern International Conference on Approximation Methods and Numerical Modelling in Environment and Natural Resources, Pau (France), June 8-11 2009

[10] Sau J., Malaterre P.-O., \& Baume J.-P. (2010) — Sequential Monte Carlo Hydraulic State Estimation of an Irrigation Canal. Compte Rendu de l'Académie des Sciences - Mécanique. 2010. ISSN 1631-0721. 338(4) 212-219

[11] Roux H., \& Dartus D. (2008) - Sensitivity analysis and predictive uncertainty using inundation observations for parameter estimation in open-channel inverse problem. J Hydraul Eng. 134(5) 541-549

[12] Pappenberger F., Beven K., Horritt M., \& Blazkova S. (2005) - Uncertainty in the calibration of effective roughness parameters in HEC-RAS using inundation and downstream level observations. Journal of Hydrology. 302 46-69

[13] Malaterre P.-O. (1998) - PILOTE : linear quadratic optimal controller for irrigation canals. ASCE Journal or Irrigation and Drainage. 124(4) 187-194

[14] Guinot V., \& Gourbesville P. (2003) - Calibration of physically based models : back to basics? Journal of Hydroinformatics. 5(4) 233-244

[15] Castaings W. (2007) - Analyse de sensibilité et estimation de paramètres pour la modélisation hydrologique : potentiel et limitations des méthodes variationnelles. Thèse de doctorat, Université Joseph Fourier

[16] Guinot V., Lemenager M. \& Cappelaere B. (2007) Sensitivity equations for hyperbolic conservation law-based flow models. Advances in Water Resources. 30(9) 1943-1961

[17] Malaterre P.-O., \& Khammash M. (2003) - 5-pool irrigation canal system. ASME Journal of Dynamic Systems, Measurement, and Control. 125(4) 639-645 